\title{
Management of bilateral rhino-orbital cerebral mucormycosis
}

\author{
Stacey C Lam *, MB, ChB, MRCSEd, Hunter KL Yuen, MRCS, FCOphthHK \\ ${ }^{1}$ Department of Ophthalmology and Visual Sciences, Hong Kong Eye Hospital, Hong Kong \\ ${ }^{2}$ Department of Ophthalmology and Visual Sciences, The Chinese University of Hong Kong, Shatin, Hong Kong \\ * Corresponding author: staceylam@gmail.com \\ Hong Kong Med J 2019;25:408-9 \\ https://doi.org/10.12809/hkmj187588
}

\section{Introduction}

Mucormycosis is a group of invasive infections caused by filamentous Mucorales fungi, most commonly Rhizopus species. ${ }^{1}$ Although rare, Mucorales is the third most common cause of fungal infection after Candida and Aspergillus species. ${ }^{2}$ Mucorales are opportunistic fungi that can be found in soil, as well as the mouth, nasal tract, and faeces of healthy individuals. Contact with Mucorales occurs through spore inhalation; the organism can then invade the paranasal sinus mucosa and erode through the bony walls of the orbit and skull base into the brain, causing orbital and cerebral infections, respectively. Fungal growth is enhanced in high-glucose, high-iron, and acidic environments. ${ }^{1}$ Moreover, elevated glucose and iron levels up-regulate glucose-regulated protein 78 (GRP78) and promote endothelial cell invasion. ${ }^{3}$ The hallmark of mucormycosis is angioinvasion, causing arteritis, vessel thrombosis, tissue ischaemia, and necrosis with bony destruction. ${ }^{1}$ Angioinvasion also allows the organism to disseminate to other organs, and the ischaemic necrosis impedes the delivery of antifungal agents to target sites.

Rhino-orbital cerebral mucormycosis (ROCM) is a rare but life-threatening fungal infection that

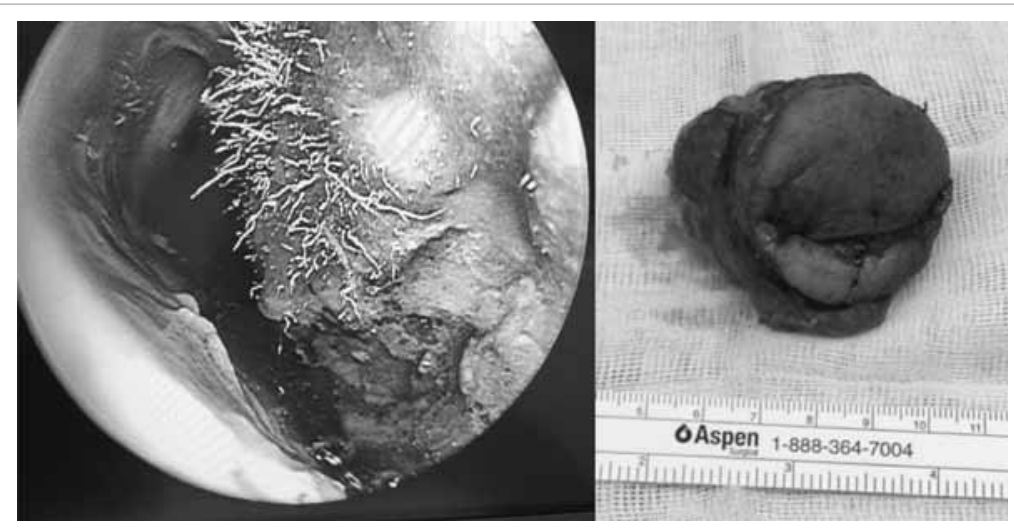

FIG. Intraoperative photo of exenterated right eye and right nasal sinus endoscopy showing Mucorales hyphae occurs in immunocompromised or diabetic patients. Bilateral involvement in ROCM has been reported in only a few cases..$^{1,4,5}$

\section{Exemplar case}

Rhino-orbital cerebral mucormycosis is usually unilateral, with the infection spreading from the nasal mucosa to the sinuses, orbit and brain. We experienced a severe case of bilateral ROCM. A 70-year-old obese woman presented with poor appetite and right eye pain for 10 days. She had a history of well-controlled type 2 diabetes mellitus (haemoglobin A1c 6.5\%, 10 months before presentation). On admission, she was febrile, with right upper lid and facial swelling, right proptosis and ophthalmoplegia. She had elevated blood glucose $(21.8 \mathrm{mmol} / \mathrm{L})$, elevated haemoglobin A1c (17.9\%), and urine test revealed ketone bodies and leukocytes. Her preliminary diagnosis was right orbital cellulitis with sinusitis, diabetic ketoacidosis, and urinary tract infection. Despite insulin infusion and hydration, aggressive antibiotics with ceftriaxone, metronidazole, and amphotericin B, the patient developed septic shock, acute coronary syndrome, frontal lobe stroke, and acute kidney injury requiring dialysis.

At 3 days after admission, the patient developed signs of right endophthalmitis and right forehead phlebitis. Urgent aqueous and vitreous tap with injection of intravitreal vancomycin and ceftazidime was performed; both cultures were negative. Nasal biopsy yielded Rhizopus oryzae and mucormycosis, and she was started on systemic liposomal amphotericin B, posaconazole and anidulafungin. Infected tissue was urgently debrided by orbital exenteration with functional endoscopic sinus surgery. Intra-operative endoscopy showed nasal hyphae (Fig). After surgery, the wound was irrigated daily with amphotericin $B$. Despite local and systemic antifungal treatment, the infection was not controlled and 1 week later, spread to the contralateral sinus, orbit, and eye with endophthalmitis. The patient succumbed 4 weeks after admission. 


\section{Discussion}

Effective treatment of ROCM includes (1) early diagnosis; (2) reversal of underlying risk factors; (3) surgical debridement where applicable; and (4) prompt antifungal therapy. ${ }^{2}$

First, early diagnosis depends on a high index of clinical suspicion. Onset of mucormycosis can be nonspecific with malaise and fever. With orbital involvement, there can be orbital cellulitis, orbital apex syndrome, and cavernous sinus thrombosis. Occurrence of mental state changes, hemiparesis, or seizures suggest intracranial extension. ${ }^{2}$ Endoscopy and radiography appearance lag behind clinical progression, so in suspicious cases, blind biopsies of sinus mucosa or thickened extraocular muscles are warranted. ${ }^{2}$ Computed tomography findings are nonspecific, with sinusitis or thickening of extraocular muscles, but may be useful in delineating the extent of the infection and in guiding surgical debridement. ${ }^{2}$ Magnetic resonance imaging is more sensitive than computed tomography for detecting orbital and cerebral involvement, and may demonstrate focal lack of enhancement with devitalised sinus mucosa. ${ }^{6}$ Definitive diagnosis is made by demonstration of fungal hyphae in tissue specimens. Fungal invasion may be patchy, so multiple biopsies may be required for definitive diagnosis. ${ }^{2}$

Second, it is critical to reverse any underlying immunocompromised state. This includes aggressive management to restore euglycaemia and normal acid base in diabetic ketoacidotic patients, stopping immunosuppressive agents, and avoiding iron and blood transfusions. ${ }^{2}$ Other therapies include iron chelating agents, hyperbaric oxygen, and adjunctive cytokine therapy. ${ }^{2,5}$

Third, surgical debridement is critical, as blood vessel thrombosis and resulting tissue necrosis impedes delivery of necessary antifungal agents to the site of infection. Orbital exenteration has been found to make a significant difference in survival only in patients with fever. ${ }^{6}$ Involved tissues rarely bleed, so surgeons should debride until well-perfused bleeding tissue is encountered. Daily repeated debridement may be needed, and subsequent surgeries may be needed for reconstruction when the infection subsides. Bilateral exenteration for bilateral mucormycosis is uncommon with poor prognosis, but survival from bilateral exenteration has been reported. ${ }^{5}$ For our case, bilateral exenteration was not attempted as the patient already had poor prognosis and the surgery would be extremely disfiguring if the patient survived.

Fourth, first-line antifungal treatment includes lipid-based amphotericin B, which destroys the cell wall of the fungus. This is given systemically, locally irrigated, and packed in affected areas. Fluconazole, voriconazole, and itraconazole do not have reliable activity against mucormycosis. ${ }^{2}$ Posaconazole, a trizole antifungal, has been suggested as a salvage therapy for those who are refractory or intolerant to polyene.

Poor survival is associated with delayed diagnosis and treatment (61\% if commenced within first 12 days of presentation compared to $33 \%$ if after 13 days), cerebral involvement (hemiparesis or hemiplegia), bilateral sinus involvement, renal disease, and possibly facial necrosis. ${ }^{7}$

Owing to the aggressive nature of mucormycosis, the mortality rate is high. Although an infrequent diagnosis, there should be a high index of suspicion for mucormycosis in patients with predisposing factors and orbital symptoms, in order to prevent treatment delay.

\section{Author contributions}

HKL Yuen contributed to the concept of study, acquisition and analysis of data, and critical revision for important intellectual content. SC Lam wrote the article.

\section{Conflicts of interest}

As an epidemiology advisor of the Editorial Board, HKL Yuen was not involved in the peer review process of the article. The other author has disclosed no conflicts of interest.

\section{Funding/support}

This research received no specific grant from any funding agency in the public, commercial, or not-for-profit sectors.

\section{Ethics approval}

The study adhered to the tenets of the Declaration of Helsinki.

\section{References}

1. Jiang N, Zhao G, Yang S, et al. A retrospective analysis of eleven cases of invasive rhino-orbito-cerebral mucormycosis presented with orbital apex syndrome initially. BMC Ophthalmol 2016;16:10.

2. Spellberg B, Edwards J Jr, Ibrahim A. Novel perspectives on mucormycosis: pathophysiology, presentation, and management. Clin Microbiol Rev 2005;18:556-69.

3. Liu M, Spellberg B, Phan QT, et al. The endothelial cell receptor GRP78 is required for mucormycosis pathogenesis in diabetic mice. J Clin Invest 2010;120:1914-24.

4. Oladeji S, Amusa Y, Olabanji J, Adisa A. Rhinocerebral mucormycosis in a diabetic case report. J West African Coll Surg 2013;3:93-102.

5. De La Paz MA, Patrinely JR, Marines HM, Appling WD. Adjunctive hyperbaric oxygen in the treatment of bilateral cerebro-rhino-orbital mucormycosis. Am J Ophthalmol 1992;114:208-11.

6. Hargrove RN, Wesley RE, Klippenstein KA, Fleming JC, Haik BG. Indications for orbital exenteration in mucormycosis. Ophthalmic Plast Reconstr Surg 2006;22:286-91.

7. Ferry AP, Abedi S. Diagnosis and management of rhino-orbitocerebral mucormycosis (phycomycosis). A report of 16 personally observed cases. Ophthalmology 1983;90:1096-104. 